\title{
New records of two Barsine species (Lepidoptera, Erebidae, Arctiinae) from Southeast Asia
}

\author{
Vitaly M. Spitsyn, ${ }^{1,2}$ Nikita I. Bolotov ${ }^{1}$
}

1 Russian Museum of Biodiversity Hotspots, Federal Center for Integrated Arctic Research, Russian Academy of Sciences, Severnaya Dvina Emb. 23, 163000 Arkhangelsk, Russia. 2 Department of Science, Northern Arctic Federal University, 163002 Arkhangelsk, Russian Federation. Corresponding author: Vitaly M. Spitsyn, vitalik91993@yandex.ru

\begin{abstract}
In this study, we report the first records of Barsine linga Moore, 1859 from Laos and Barsine maculifascia (Hampson, 1894) from Vietnam.
\end{abstract}

Key words

Biogeography; biodiversity; Barsine linga; Barsine maculifascia; Laos; Vietnam.

Academic editor: Reza Zahiri | Received 16 January 2018 | Accepted 16 February 2018 | Published 16 March 2018

Citation: Spitsyn VM, Bolotov NI (2018) New records of two Barsine species (Lepidoptera, Erebidae, Arctiinae) from Southeast Asia. Check List 14 (2): 353-355. https://doi.org/10.15560/14.2.353

\section{Introduction}

According to our previous works, the geographic distribution of Asian tropical tiger moths insufficiently studied (Spitsyn et. al. 2015, 2016). In the literature, checklists on the fauna of several countries, such as Thailand (Cerny and Pinratana 2009), Malaysia (Bucsek 2012, 2014), China (Fang 2000), and India (Kirti and Singh 2016) are available. However, information on whether species of tiger moths are present in other parts of Southeast Asia, such as Myanmar, Laos, Cambodia, and partially Vietnam, remains poorly available. In the present paper, we document new data on the geographic distribution of 2 species of Lithosiini (Erebidae, Arctiinae) in the genus Barsine Walker, 1854.

\section{Methods}

The specimens were collected using 2 UV lamps: Medium 250 WSB/E24-E23 250 Watt Self Ballast Mer- cury VaporClear and F15W/T8/Quantum Black Light, Germany. The genitalia were dissected according to a standard approach, which was described in our previous paper (see Spitsyn et. al. 2016). Images of specimens were taken with a camera (Canon EOS 650D with Canon EF 100mm f/2.8L Macro IS USM). Photographs of genitalia were taken with a stereomicroscope (SteREO Discovery.V8, Carl Zeiss, Germany). The base map used is from http://alabamamaps.ua.edu/contemporarymaps/ world/asia/index 2 .html.

The specimens were deposited in the collection of the Russian Museum of Biodiversity Hotspots, Federal Center for Integrated Arctic Research, Russian Academy of Sciences (Russian Federation).

\section{Results}

For the first time, we present records of Barsine linga Moore, 1859 from Laos and Barsine maculifascia Moore (Hampson, 1894) from Vietnam. 

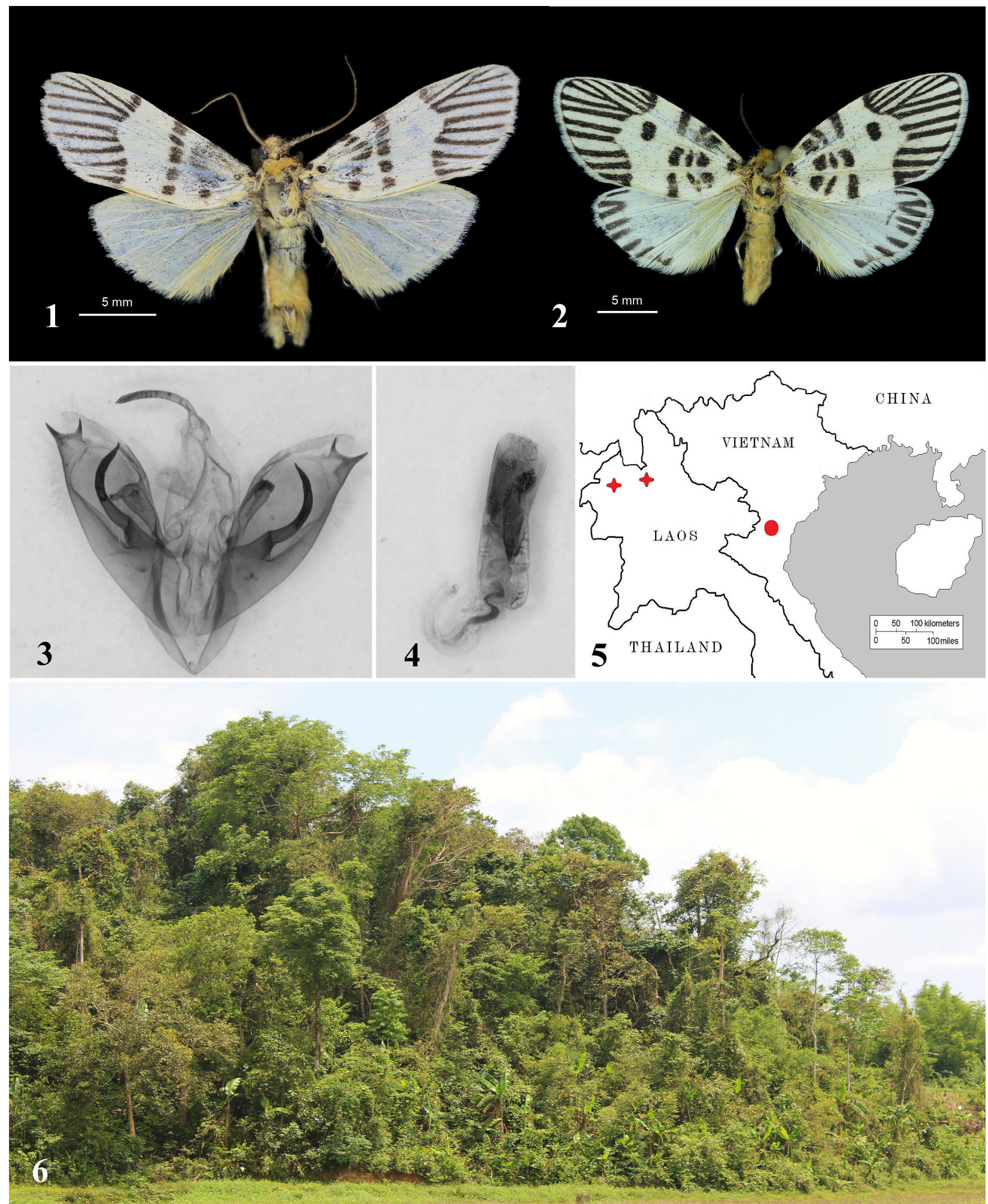

Figures 1-6. 1. Male Barsine linga (dorsal view). 2. Female B. maculifascia (dorsal view). 3. Male genitalia of $B$. linga. 4. Aedeagus of $B$. linga. 5. Map of new records of B. linga (red asterisks) and B. maculifascia (red circle). 6. Habitat of B. linga in Laos (Luang Namtha Province, Viangphoukha town, tropical forest) (Photographs: Vitaly M. Spitsyn).

\section{Barsine linga Moore, 1859}

Figures 1, 3-6

Material examined. Laos, Oudomsay Province, Oudomsay city, damaged forest on border of the city, $20^{\circ} 39.772$ N, 101 $58.233^{\prime}$ E, 1 ô, 01.05-03.05.2016, Spitsyn leg. (RMBH: voucher no. Sph0730); Laos, Luang Namtha Province, Viangphoukha town, tropical forest, $20^{\circ} 40.972^{\prime}$
N, 10104.692' E, 1 गे, 24.05-25.05.2016, Spitsyn leg. (RMBH: voucher Sph0704).

Diagnosis. This species looks very similar to $B$. maculifascia (Hampson, 1894), B. pluma Cerny 2009, and B. syntypica Swinhoe, 1906 but differs by a lack of a discal black spot on the forewing. 
Distribution. India: Sikkim; Nepal, China: Yunnan; Thailand (Fang 2000, Cerny and Pinratana 2009) and Laos.

\section{Barsine maculifascia (Hampson, 1894)}

Figures 2, 5

Material examined. Vietnam, Thanh Hoa Province, Tan Thanh village, plants on sides of rice fields, $19^{\circ} 37.788^{\prime}$ $\mathrm{N}, 105^{\circ} 18.505^{\prime} \mathrm{E}, 29.03 .2016,2$ \%, Spitsyn leg. (RMBH: vouchers Sph0702 and Sph0703).

Diagnosis. This species differs from B. pluma and B. syntypica by its larger body size. The wingspan is $30-33 \mathrm{~mm}$ (our data, Bucsek 2014), while the wingspan of B. pluma and $B$. syntypica is $18-22 \mathrm{~mm}$ (Bucsek 2014). Unlike $B$. linga, this species has a discal black spot on the forewing.

Distribution. Myanmar (Burma), China: Yunnan, Guangxi; Thailand, Malaysia (Barow 1982, Fang 2000, Cerny and Pinratana 2009, Bucsek 2014) and Vietnam.

\section{Discussion}

In conclusion, we can assume that the geographic distribution of Barsine linga includes areas from the foothills of the Himalayas in northern India to Nepal. In the east, it the range extends south of Yunnan province and territories of Laos and north-west Thailand. It seems that this species inhabits humid tropical forests in the mountains. Accordingly, it is highly possible that the species will be discovered in the states of Kachin and Shan in Myanmar. The geographic range of B. maculifascia extends to the Pacific coast of the Indochina Peninsula. We assume that this species should also be in Laos, because it was found near the border of this country.

\section{Acknowledgements}

We would like to thank Dr Nhi Thi Pham for her valuable help during the fieldwork. Our sincerest gratitude also goes to Bai Yunfeng and Feng Liu for their support in translating Chinese literature. This study was partly supported by the Federal Agency for Scientific Organizations (project no. 0409-2015-0143).

\section{References}

Barow HS (1982) An Introduction to the Moths of South East Asia. The Malayan Nature Society, Kuala Lumpur, 305 pp.

Bucsek K (2014) Erebidae, Arctiinae (Lithosiini, Arctiini) of Malay Peninsula-Malaysia. Supplementum. Institute of Zoology SAS, Bratislava, $42 \mathrm{pp}$.

Bucsek K (2012) Erebidae, Arctiinae (Lithosiini, Arctiini) of Malay Peninsula-Malaysia. Institute of Zoology SAS, Bratislava, $170 \mathrm{pp}$.

Cerny K \& Pinratana A (2009) Moths of Thailand. Vol. 6. Arctiidae. Brothers of Saint Gabriel in Thailand, Bangkok, 283 pp.

Fang C (2000) Fauna Sinica Insecta. Vol. 19. Lepidoptera: Arctiidae. Science Press, Beijing, 589 pp.

Kirti JS, Singh N (2016) Arctiid Moths of India. Vol. 2. Nature Books India, New Delhi, 214 pp.

Spitsyn VM, Bolotov IN, Gofarov MY, Bolotov NI (2016) First record of the genus Aethalida Walker, 1865 (Lepidoptera: Erebidae: Arctiinae) from Flores Island, East Nusa Tenggara, Indonesia. Ecologica Montenegrina 6: 56-60.

Spitsyn VM, Bolotov IN, Gofarov MY, Vikhrev IV, Bolotov NI (2015) First record of Nyctemera adversata (Schaller, 1788) and N. carissima (Swinhoe, 1891) (Lepidoptera: Erebidae: Arctiinae) in Myanmar. Check List 11 (4): 1687. https://doi.org/10.15560/11.4.1687 